\title{
Hazard of deceptive advertising of athletic footwear
}

\author{
Steven Robbins, Edward Waked
}

\begin{abstract}
Objectives-Athletic footwear are associated with frequent injury that are thought to result from repetitive impact. No scientific data suggest they protect well. Expensive athletic shoes are deceptively advertised to safeguard well through "cushioning impact", yet account for $123 \%$ greater injury frequency than the cheapest ones. This study tested the hypothesis that deceptive advertising creates a false sense of security with users of expensive athletic shoes, inducing attenuation of impact moderating behaviour, increased impact, and injury.
\end{abstract}

Methods-Fifteen young healthy male volunteers confronted four surfaces: a bare force moment platform, and three with this platform covered by identical shoe sole material made to appear different and advertised divergently. Advertising messages suggested superior impact absorption and protection (deceptive message), poor impact absorption and high injury risk (warning message), and unknown impact absorption and safety (neutral message). Ground reaction forces were recorded for 10 barefoot footfalls, according to a protocol requiring stepping forward from perch to a surface $4.5 \mathrm{~cm}$ below.

Results-Impact varied as a function of advertising message $(p<0.001)$. Deceptive message equalled neutral message in eliciting higher impact than the warning message and the bare platform. Differences grew with repetitions $(p<0.001)$.

Conclusions-These data provide a plausible mechanism explaining higher injury frequency in users of expensive athletic shoes. This is the first report to suggest: (1) deceptive advertising of protective devices may represent a public health hazard and may have to be eliminated presumably through regulation; (2) a tendency in humans to be less cautious when using new devices of unknown benefit because of overly positive attitudes associated with new technology and novel devices.

(Br F Sports Med 1997;31:299-303)

Keywords: sports injuries; athletic footwear; impact; running; advertising; product safety; warning labels
Research dealing with product advertising has focused on consumer preferences-it is not known if advertising influences how consumers use products after purchasing them. Advertising tends to be persuasive when dealing with opaque attributes but less effective when promoting easily appreciated properties. Safety provided by products designed to protect is usually obscure to users, therefore advertising products as offering improved safety has the potential of being convincing.

We consider advertising of protective devices to be truthful when the message suggests protection consistent with data from objective product testing under conditions of use before or after marketing of a product, whereas deceptive advertising implies protection significantly exceeding equivalent data. Suggesting good protection against injury may convince the user that cautious behaviour previously exercised is less necessary. Attenuated caution could affect safety differently when dealing with products that are either truthfully or deceptively advertised. Protective equipment proved capable of protecting before advertising, and truthfully advertised as effective, may prove less beneficial or perhaps even ineffective once safety benefits are disclosed to the public, because users may conclude that caution previously exercised is less necessary. Products deceptively advertised as performing well, that are actually ineffective, might attenuate caution sufficiently to heighten injury frequency above levels existing before their introduction.

Protective athletic equipment represents a vast group of items. Perhaps no item in this group is advertised as extensively as athletic footwear, which are all promoted as capable of "cushioning" impact via sole materials. Impact amplitude, which can be measured objectively with precision, is poorly appreciated by humans when they wear athletic footwear, ${ }^{2}$ therefore advertising this attribute presumably would be convincing.

No athletic footwear, regardless of manufacturer and price range, have ever been shown to protect well against injuries, therefore advertising good protection is deceptive. Expensive footwear are subject to extremely deceptive advertising. They are advertised to improve protection over cheaper products by 
incorporating new features that protect, and more advanced safety technology, yet epidemiological data indicate that users of more expensive shoes are injured more frequently. Expensive shoes accounted for $123 \%$ greater frequency of injury than the lowest cost models, regardless of manufacturer, after correcting for previous injury, training mileage, and sex, factors influencing injury frequency. ${ }^{3-5}$ This suggests that impact is greater when consumers use more expensive athletic shoes, and we can think of nothing other than the effect of advertising that might account for it.

As an explanation of the higher injury frequency when humans use expensive athletic shoes, we hypothesise that deceptive advertising suggesting good protection against impact persuades users to eschew cautious behaviour, resulting in higher impact. We tested this in an experiment in which the independent variable was the advertising message deceptively describing the same shoe sole material made to appear different as either effective protection, unsafe, or of unknown safety. The dependent measure was impact.

\section{Methods}

SUBJECTS

A random sample of 15 healthy young men with a mean (SD) age of 31 (4.2) years participated in this study on a voluntary basis. All were selected from the general population and participated actively in sports and leisure activities. Mean (SD) body mass and height were $75(9.2) \mathrm{kg}$ ) and $175(7.8) \mathrm{cm}$, respectively. All were free of conditions affecting their ability to walk, run or balance.

SOLE INTERFACE MATERIAL

The sole interface material covering the top surface of the force moment platform consisted of a $2.5 \mathrm{~cm}$ thick layer of ethyl-vinyl acetate (EVA), Shore A10 in hardness. EVA is the polymer material most commonly used in footwear soles. Sole conditions differed only in the colour of the fabric encasing the EVA sole material. This envelope ensured subjects could not identify the material within it.

\section{MESSAGES FOR SHOE SOLE ADVERTISING}

Deceptive message

This message resembled advertisements used to promote athletic footwear in periodicals available to us. It included pseudoscientific graphs and tables, and athlete endorsement suggesting superior impact absorption and stability. We used the terms "advanced technology" and "state of the art", to describe the sole. We stated that this technology is incorporated in the most expensive athletic shoes currently on the market.

\section{Warning message}

This message was based on pre-experimental evaluation of user opinions, indicating that users believed that athletic shoes were effective in protecting against injury, that expensive shoes protected better than cheap ones, and that new technology has resulted in more pro- tective shoes. We concluded that contrasting notions from the deceptive message would constitute warning. The shoe sole was described as associated with frequent injuries from excessive impact and instability. Subjects were told that this material was old technology and used only in cheap running shoes.

\section{Neutral message}

This shoe sole material was described as never used in shoe soles previously, and impossible to predict how it might influence either impact absorption or stability.

FORCE MOMENT PLATFORM

A unique force moment platform was used. This force moment platform is $500 \mathrm{~mm}^{2}$ in area and $150 \mathrm{~mm}$ high. It is capable of measuring peak loads of up to $10000 \mathrm{~N}$ with a resolution of $1 \mathrm{~N}$ for forces and $0.1 \mathrm{Nm}$ for moments. The maximum acquisition/ transmission rate for each load sensor is $500 \mathrm{~Hz}$. A high speed (115 kbaud) serial link transmits data from force moment platform to host computer, which calculates various performance indices and records data to disk. A graphical user interface allows operator to view time history of the forces, moments, and centre of force.

\section{TESTING PROTOCOL}

Written consent was obtained both before beginning the experiment and followed the ethical guidelines for human experimentation set by the Helsinki Declaration. Subjects were confronted with four surface interface conditions in a counterbalanced order: a bare rigid force moment platform, and three with this platform covered by identical impact absorbing shoe sole material. The advertising messages were presented in writing and graphics. Before each surface condition, the advertising message was read by the subject and re-read by the technician. No message was presented for the bare force moment platform. Impact testing consisted of 10 consecutive barefoot footfall impacts recorded on the force moment platform for each surface condition. Subjects were required to step forward from perch to a surface $4.5 \mathrm{~cm}$ below, and land on one foot, as shown in figure 1 . Subjects were debriefed at the end of the experiment to assess any subject bias regarding the experiment and its protocol. Included in the debriefing were questions concerning the credibility of claims made by manufacturers of modern athletic shoes concerning protection against injuries, and whether subjects were aware of any reports or advertisements contrary to these claims.

\section{DATA ANALYSIS}

One way analysis of variance was performed for each dependent measure using the three material conditions as independent variables. Post hoc tests were performed for significant $F$ values only. Differences between the bare platform surface condition and the three other material surface conditions were assessed using $t$ test with Bonferroni adjustment to correct for type I error. Statistical significance for all tests was set at 0.05 . 

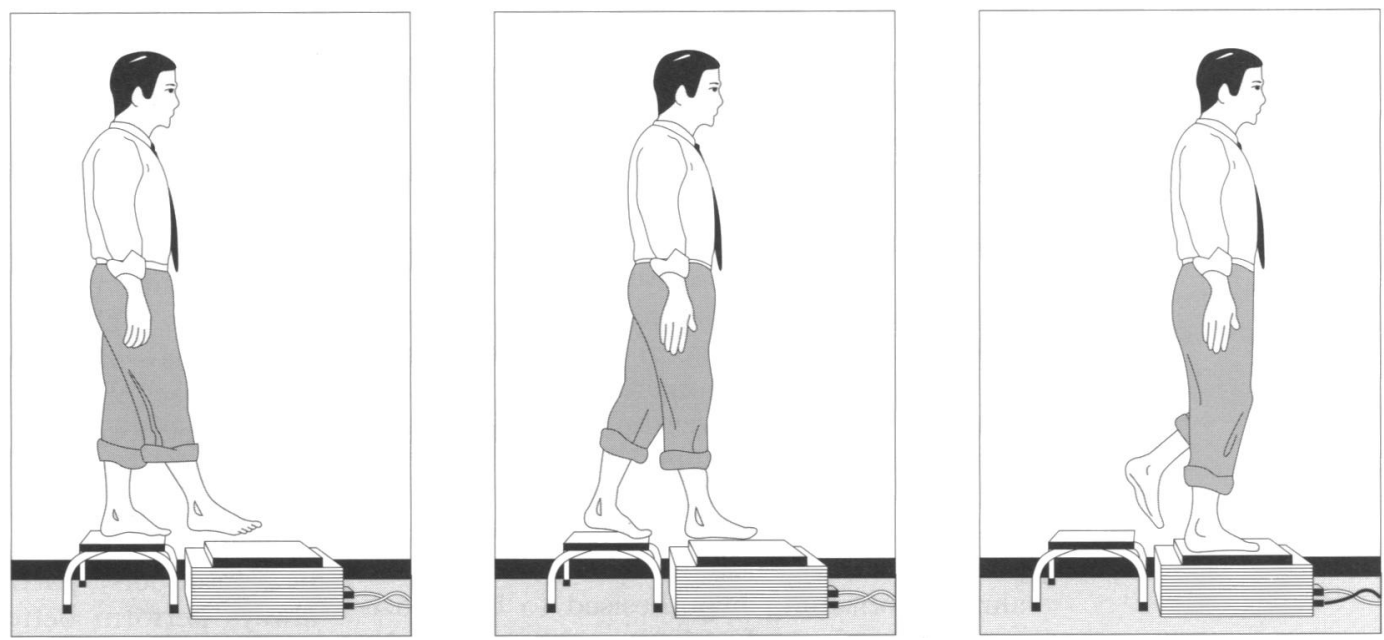

Figure 1 Schematic representation of experimental set up for dynamic footfall testing. Subject stepped down onto force moment platform, which was either bare, or covered by athletic shoe sole material. He balanced on one foot after foot contact with platform. Subjects were barefoot with gaze straight and arms to side.

\section{Results}

Impact varied in relation to advertising claims $(F(2,28)=96.87, \mathrm{p}<0.001)$. Impact was greatest with the deceptive message and lowest with the warning message, with means of $121 \%$ and $110 \%$ body weight (BW), respectively. Neutral message, with a mean impact of $117 \% \mathrm{BW}$, did not significantly differ from the deceptive message, yet was significantly greater than the warning message. Mean impact with the bare force moment platform was $108 \% \mathrm{BW}$ (fig 2) This was significantly lower than the deceptive and neutral messages, but not significantly lower than the warning message. There was a

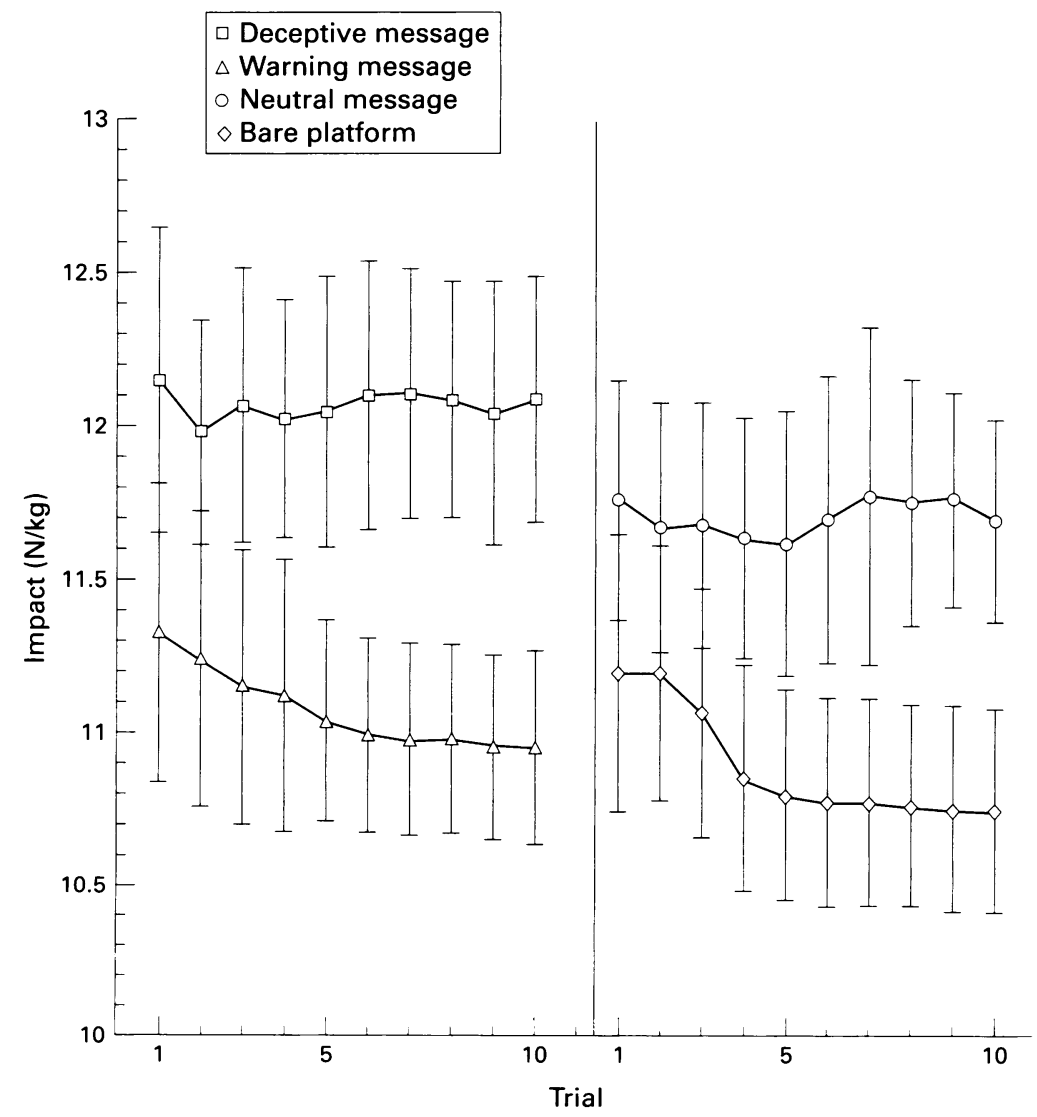

Figure 2 Mean footfall impact values with standard error bars for the 10 consecutive trials for each of the three surface interface conditions and the rigid bare platform. Footfall impact values stabilised after the fifth trial, except for the rigid platform, which stabilised after the third trial. significant interaction effect between message and trial $(F(18,252)=5.16, \mathrm{p}<0.001)$. Post hoc tests indicated that with each subsequent trial, footfall impact significantly increased with the deceptive message, and significantly decreased with warning message, yet no change occurred with the neutral message.

\section{Discussion}

The notion that cumulative microtrauma from impact experienced during running and jumping causes injury in humans is supported by epidemiological reports indicating that every sport involving running is associated with frequent lower extremity injury,,$^{5-8}$ and experimental studies showing that in animals, repetitive microtrauma from impact of locomotion accounts for injury in relation to cumulative exposure. ${ }^{910}$ Humans are capable of moderating impact dramatically through behaviours, such as knee and hip flexion at contact of support surface. ${ }^{11-14}$ Amplitude of impact when gymnasts land impulsively has been shown to be directly related to hip and knee flexion amplitude. ${ }^{11} 15$

Attempts at moderating excessive impact during running and jumping through external devices, such as yielding interfaces (for example, soft athletic footwear and mats), has produced disappointing results. Impact has been shown to be higher when gymnasts land on mats than on hard surfaces, because humans greatly reduce flexion when landing on soft mats. ${ }^{11}{ }^{15}$ It is not clear if impact in humans is higher when wearing soft soled athletic footwear than when barefoot, although it is not significantly lower. This is because humans reduce flexion when soft soled shoes are used thereby at least neutralising any advantage that yielding materials offer in terms of impact absorption. ${ }^{211} 1516$ It is now believed that humans land rigidly when soft materials are underfoot in an attempt to counter the instability that these interfaces induce. ${ }^{17}$ There is no evidence that athletic footwear differ significantly in impact absorption in relation to manufacturer, model, or cost, when tested blindly using force moment platform or accelerometer technology during normal running. ${ }^{16}$ 
This finding is expected as all incorporate soles made of similar materials, and produce comparable impact enhancing instability. ${ }^{18-21}$ There are no data supporting the notion that athletic footwear are capable of protecting against injury through cushioning of impact.

Without any significant difference in impact absorption between footwear, one would anticipate that users of athletic footwear would experience no difference in injury frequency in relation to model and manufacturer. It comes at some surprise however that Marti found that expensive shoes accounted for $123 \%$ greater injury frequency than lowest cost models, regardless of manufacturer, after correcting for previous injury, training mileage, sex, and almost everything hypothesised to be associated with injury. ${ }^{5}$ Furthermore, runners who expressed strong brand preference had a significantly greater chance of being injured than those who had no brand loyalty. This suggests that footwear products may be used differently in relation to cost based on expectations of protection as communicated through advertising. As an explanation of injury frequency in relation to athletic footwear cost, we hypothesised that advertising affects user caution, thereby inducing users to adjust impact moderating behaviour in relation to perceived risk inferred by the advertising message- - higher impact with expensive models that are assumed to be safe, and lower impact with inexpensive models that are presumed dangerous.

In this experiment impact amplitude varied as a function of advertising message, with the warning message resulting in lower impact than the deceptive one, $110 \% \mathrm{BW}$ versus $121 \%$ $\mathrm{BW}$, respectively $(\mathrm{p}=0.031)$. With repetitive footfalls, impact amplitude declined and increased in relation to the warning and deceptive message, respectively ( $p<0.001)$. This supports our hypothesis that footwear advertising changes usage behaviour along lines accounting for higher injury frequency with more expensive shoes and lower injury frequency with inexpensive shoes.

The bare platform resulted in lowest impact of all conditions, though not significantly different from the warning message (bare, $109 \% \mathrm{BW}$; warning, $110 \% \mathrm{BW}, \mathrm{p}=0.15$ ). During debriefing subjects reported that landing on the bare platform was uncomfortable, whereas the sensation associated with landing on any of the sole conditions was more pleasant. As the warning message was equivalent to painful consequences in inducing impact moderating behaviour, we conclude that use of warning messages is a highly effective means of eliciting cautious behaviour.

The deceptive message resulted in highest impact, though not significantly greater than with the neutral message (deceptive, $121 \%$ BW; neutral, $117 \% \mathrm{BW}, \mathrm{p}=0.16$ ). Equivalency of neutral and deceptive message is explained by information obtained during debriefing of subjects. Higher impact with the deceptive message is consistent with our hypothesis that attenuated caution is based on a false sense of security resulting from deceptive advertising messages. This is further aided by pre-existing beliefs subjects reported during debriefing that associates protection against injury with expensive footwear and little or no protection with cheaper ones, notions based on previous deceptive advertising of athletic footwear and false information disseminated by retail outlets. In addition, many subjects harboured false impression about truth in advertising regulations-most believed companies were required to present a truthful assessment of product performance in advertisements.

We believe that higher impact with neutral message is accounted for by general irrational faith in new products. Subjects believed that new products, particularly safety products, always perform better than older ones. This positive attitude about novelty seemed based in part on the mistaken impression that government regulations require all new safety products to be tested for proof of satisfactory performance under conditions of use before being available to the public. In addition, some positive notions about new athletic footwear seem to be generalisations based on brand loyalty, which in turn was based on deceptive advertising of other footwear products. This is understandable considering that humans can be easily mislead by advertising that deals with product attributes that are opaque to users. ${ }^{122} 23$

Practical application of our data relate to two conditions: protective devices truthfully and deceptively advertised. This report is the first to imply that safety products truthfully advertised to protect effectively before being commercialised might be less effective than anticipated after commercialisation, because of a strong tendency in humans to become less cautious when they use products they believe protect better. This is also the first report to suggest that deceptive advertising of protective devices is a clear public health hazard, because humans will become less cautious and may injure themselves more frequently when they use them. We believe diminished vigilance caused by this advertising accounted for $123 \%$ greater injury frequency in users of expensive running shoes that Marti reported, and probably continues to be responsible for innumerable injuries. ${ }^{5}$ Deceptive advertising of protective devices must be eliminated.

Our report suggests that a simple and direct solution to this tendency in humans to be less cautious when using new safety products lies in adequate warning labelling, as we found that warning message was highly effective in eliciting cautious behaviour in this experiment. Public health would be advanced if truthfully advertised protective devices possess a prominent explicit warning label affixed to them explaining to users that protection against injury with this product is only realisable if they remain as cautious with the new product as they were with the one it replaced. The results of warning labelling may be impressive, considering with respect to expensive running shoes, we estimate that this labelling could result in an injury frequency reduction of $55 \%$. 
One limitation of this experiment is that experimental conditions differed from natural conditions-stepping off a perch differs from how footwear products are used during locomotion. Shock absorbing materials have been shown to influence impact similarly whether tested by humans stepping from a perch or during actual locomotion. For example, McNitt-Gray found during landings from a platform, ${ }^{15}$ and Nigg during running, ${ }^{16}$ that impact increases as interfaces become softer. This suggests that this limitation should not influence our conclusions.

In summary, pre-existing beliefs of protection against injury formed through deceptive advertising of athletic footwear attenuates user caution thereby amplifying impact. This may explain the $123 \%$ higher injury frequency associated with more expensive shoes. ${ }^{5}$ Accordingly, deceptive advertising of protective devices is a public health hazard and must be eliminated. Humans are less cautious even when they use truthfully advertised products because of excessively positive attitudes toward new products and wrong impressions of the standards of truth in advertising through government regulation.

1 Snyder R. Misleading characteristics of implied superiority claims. Fournal of Advertising 1989;18:54-61.

2 Robbins SE, Hanna AM, Jones LA. Sensory attenuation induced by modern athletic footwear. fournal of Testing and Evaluation 1988;16:412-6.

3 Gross TS, Bunch RP. Material moderation of plantar impact stress. Med Sci Sports Exer 1989;21:619-24.

4 Hamill J, Bates BT. A kinetic evaluation of the effects of in-vivo loading on running shoes. $\mathcal{F}$ Orthop Sports Phys Ther 1988;10:47-53.

5 Marti B. Relationship between running injuries and running shoes - Results of a study of 5000 participants of a $16-\mathrm{km}$ run - The May 1984 Berne "Grand Prix". In: Segesser B, Pforringer W, eds. The shoe in sport. Chicago: Year Book Medical Publishers, 1989: 256-65.
6 Brunet ME, Cook SD, Brinker MR, Dickson JA. A survey of running injuries in 1505 competitive and recreational runrunning injuries in 1505 competitive and recrea

7 Clement DB, Taunton JE, Smart GW, McNicol JL. A survey of overuse running injuries. Physician Sportsmed 1981;9:4758.

8 James SL, Bates BT, Osternig LR. Injuries to runners. $A m \mathcal{f}$ Sports Med 1978;6:40-50.

9 Dekel S, Weissman SL. Joint changes after overuse and peak overloading of rabbit knees in vivo. Acta Orthop Scand 1978;49:519-28.

10 Hellman DB, Helms CA, Genant HK. Chronic repetitive trauma: a cause of atypical degenerative joint disease. Skeletal Radiol 1983;10:236-42.

$11 \mathrm{McNitt-Gray} \mathrm{JL,} \mathrm{Yokoi} \mathrm{T.} \mathrm{The} \mathrm{influence} \mathrm{of} \mathrm{surface} \mathrm{charac-}$ teristics on the impulse characteristics of drop landings. Proceedings of the $13^{\text {th }}$ Annual Meeting of the American Society of Biomechanics. Vermont: American Society of Biomechanics, 1989: 92-3.

12 Robbins SE, Hanna AM. Running related injury prevention through barefoot adaptations. Med Sci Sports Exer 1987;19: 148-56.

13 Robbins SE, Hanna AM, Gouw GJ. Overload protection: avoidance response to heavy plantar surface loading. Med Sci Sports Exer 1988;20:85-92.

14 Robbins SE, Hanna AM, Gouw GJ. Running related injury prevention through innate impact moderating behaviour. Med Sci Sports Exer 1989;21:130-9.

$15 \mathrm{McNitt-Gray} \mathrm{JL.} \mathrm{Landing} \mathrm{strategy} \mathrm{adjustments} \mathrm{made} \mathrm{by}$ female gymnasts in response to drop height and mat composition. F Appl Biomech 1991;9:173-90.

16 Nigg B. Biomechanics of running shoes. Chicago: Human Kinetics Publishers, 1986.

17 Robbins S, Waked EG. Humans amplify impact to compensate for instability caused by shoe sole materials. Arch Phys Med Rehabil 1997;78:463-7.

18 Cook SD, Kester MA, Brunet ME. Shock absorption characteristics of running shoes. Am F Sports Med 1985;13:24853.

19 Robbins SE, Waked EG, Gouw GJ, McClaran J. Athletic footwear affect balance in men. $\mathrm{Br} f$ Sports Med 1994;28:117-22

20 Robbins SE, Gouw GJ, McClaran J. Shoe sole thickness and hardness influence balance in older men. $\mathcal{f}$ Am Geriatr So 1992;40:1089-94.

21 Robbins SE, Waked EG, Allard P, Krouglicof N, McClaran J. Aging in relation to optimization of footwear in older men. 7 Am Geriatr Soc 1997;45:61-7.

22 Marks LJ, Kamins MA. The use of product sampling and advertizing: effects of sequence of exposure and degree of advertizing claim exaggeration on consumers' belief strength, belief confidence, and attitudes. Fournal of Market strength, belief confidence,

23 Smith RR. Integrating information from advertizing and trial: process and effects on consumer response to product information. Fournal of Market Research 1993;30:204-19. 